\title{
Workshop on the Nonlinear Dynamics of Chemical Reactions, Processes, and Reactors Cochairmen: Yu. D. Tret'yakov and I. V. Melikhov
}

DOI: $10.1134 / \mathrm{S} 004057951006014 \mathrm{X}$

The regular workshop on the nonlinear dynamics of chemical reactions, processes, and reactors, held on June 10, 2010, was devoted to the role of cooperative interactions in a multitude of crystals and to their effect on the formation of crystalline phases. Fundamental problems of data processing and interpretation were also discussed.

Professor P.P. Fedorov, Doctor of Chemistry, Prokhorov General Physics Institute, Russian Academy of Sciences, presented a report entitled "Nonlinear Phenomena in the Agglomeration Growth of Crystals." He reported experimental data on the evolution of nanoparticle ensembles and presented evidence of crystal growth via an unconventional mechanism, specifically, cooperative consolidation of nanoparticles into microcrystals. Specifically, he reported on the preparation of nanopowders of yttrium and magnesium oxides and those of alkaline-metal fluorides containing rare-earth elements by chemical precipitation of poorly soluble compounds from aqueous solutions followed by heat treatment of the precipitate. The nanopowders were examined by scanning and transmission electron microscopy, atomic force microscopy, and X-ray diffraction. X-ray diffraction data were used to calculate the coherent scattering domain (CSD) size and the microdistortion value. It was established that the heat treatment of the precursor is accompanied by a manifestation of the shape memory phenomenon: the resulting nanoparticles of cubic yttrium oxide or magnesium oxide retain the nonequilibrium platelike shape inherited from the precursor. Upon heating, the plates of nanometer thickness break down into spheroidal nanoparticles with a narrow size distribution.

Isothermal heat treatment of an ensemble of these nanoparticles abruptly increases the X-ray CSD size by one order of magnitude. A further increase in the particle size was observed later, accompanied by the appearance of crystal faces.

Similar phenomena were revealed by shape and size analysis of fluoride nanoparticles. It was demonstrated by electron microscopy that spherical fluoride nanoparticles aggregate at room temperature into irregularly shaped intergrowths whose surface exposes the initial nanoparticles.
Discussing this report, participants of the workshop expressed keen interest in the stepwise increase in the $\mathrm{X}$-ray CSD domain size, which was evidence of an abrupt increase in the size of the microparticles. O.A. Shlyakhtin noted that similar phenomena are observed in other systems, including metals. It was hypothesized that the system undergoes self-organization via the chain nanocrystal orientation process, which is possible due to the mobility of part of the nanocrystals in their aggregates. If this is the case, then first-order phase transitions in the orientation space take place in the systems examined (P.P. Fedorov, I.V. Melikhov).

Great interest was attracted by the identification of separate and aggregated particles by various methods, including X-ray diffraction and electron microscopy (P.E. Kazin, E.A. Gudilin). P.P. Fedorov discussed the possibility of deriving reliable information about the geometrical particle size from X-ray diffraction data on CSD size and microdistortion. He pointed out that, based on these data, it is impossible to conclude unambiguously whether the scattering domain is a separate particle or this particle is included in an aggregate (V.N. Rudin). Attention was paid to the difficulties in the determination of the composition of individual particles and in the assignment of a nanoparticle to a particular solid compound. It was noted that a particle of any size that can move as a whole in space and is sufficiently stable for its size to be measurable should be treated as a particle of a new phase if it belongs to a set of particles whose size distribution function (whether discrete or continuous) is characteristic of this phase (V.P. Zlomanov, I.V. Melikhov).

Concluding the workshop, I.V. Melikhov noted that any forming substance passes through a nanostate and "remembers" it, and the problem of obtaining nanodisperse materials with preset properties is actually the problem of realizing different routes of evolution of solid substances. Investigation of the distribution of particles with respect to their properties could provide invaluable information on the given subject.

V.E. Bozhevol'nov, E.D. Kozlovskaya 